\title{
Modos de representación en los distritos: ¿diputados al servicio de los partidos o de los electores? Los casos de Chile y Bolivia
}

\author{
Mikel Barreda Díaz, ${ }^{*}$ Leticia María Ruiz Rodríguez**
}

Perfiles Latinoamericanos, 25(50)

2017 | pp. $155-177$

DOI: $10.18504 / \mathrm{pl} 2550-008-2017$

\begin{abstract}
Resumen
Este trabajo aporta evidencia empírica sobre una cuestión poco investigada en América Latina: los modos de representación. Se analiza en particular un posible dilema del parlamentario en su actividad de representación: anteponer los intereses de su partido o los de los ciudadanos de su distrito. Para el estudio empírico, se recurrió a una encuesta a asesores parlamentarios de los principales partidos bolivianos y chilenos. Los argumentos fundamentales del análisis son dos. Primero, si bien la preferencia general entre los diputados es priorizar los intereses del distrito por encima de los del partido, hay diferencias significativas entre los diputados bolivianos y chilenos. Segundo, el análisis exploratorio muestra la naturaleza multicausal de las preferencias respecto a los intereses de representación. Se constata la influencia de un grupo diverso de factores relacionados con las características del diputado, del partido y del entorno socioeconómico e institucional.
\end{abstract}

\begin{abstract}
This paper provides empirical evidence on a topic that has been hardly analyzed in Latin America: representatives modes of representation. It specifically focuses on whether representatives promote party interests or constituency interests. Data have been obtained from surveys applied to representatives and political advisers from Chilean and Bolivian main political parties. Two are the key arguments. First, despite representatives' generalized preference for defending district interests' more than party interests, significant differences between Bolivian and Chilean deputies are found. Second, exploratory analysis shows the multicausal nature of representatives' interests, including variables related to representatives, their parties as well as their socio-economic and institutional environment.
\end{abstract}

Palabras clave: modos de representación, actividad distrital, parlamentarios, Chile, Bolivia. Keywords: Modes of representation, district activity, congressmen, Chile, Bolivia.

* Doctor en Ciencias Políticas y Sociología por la Universidad de Deusto. Profesor Agregado de Ciencia Política de la Universitat Oberta de Catalunya (UOC) | mbarreda@uoc.edu

** Doctora en Ciencia Política por la Universidad de Salamanca (USAL), máster en Ciencia Política por la University of North Carolina en Chapel Hill. Profesora Titular de la Facultad de Ciencias Políticas y Sociología de la Universidad Complutense de Madrid | leticiamaria.ruiz@cps.ucm.es 


\section{Introducción ${ }^{1}$}

La centralidad de la representación en los sistemas políticos modernos ha generado una ingente masa de estudios orientados por preguntas y enfoques diversos. Los sistemas políticos de Latinoamérica no han sido la excepción. Así lo confirma un simple vistazo a las revistas académicas que publican trabajos sobre esta región. No obstante, son muchas las cuestiones relativas a los procesos de representación latinoamericanos de las que se tiene un conocimiento limitado. Una de ellas se refiere a los modos de representación, esto es, a la forma en que los parlamentarios ejercen su labor de representación. A diferencia del seguimiento que ha recibido esta cuestión en Europa y Estados Unidos, en América Latina ha tenido poca atención. Este trabajo, que se ocupa de los diputados de Bolivia y Chile, aporta evidencia empírica que contribuye a ampliar el conocimiento sobre el asunto. En particular, el artículo se enfoca en la actividad de los diputados en los distritos. Se trata de uno de los ámbitos en los que discurre su tarea de representación y que en América Latina apenas ha sido examinado. Nos ocupará una dimensión concreta de los modos de representación: la priorización de los diputados entre la defensa de intereses del partido y los intereses de los ciudadanos del distrito.

El artículo se propone responder a dos preguntas. En primer lugar, ¿̇hay diferencias significativas en la priorización de intereses de representación entre los diputados chilenos y los bolivianos? Aunque en su tarea de representación combinen ambas estrategias, se trata de ver si los diputados tienden a comportarse como servidores de los partidos o de los ciudadanos. En segundo lugar, si hay diferencias, ¿qué factores pueden explicarlas? Se espera encontrar variaciones relevantes en las preferencias respecto a los intereses de representación y que en estas variaciones tengan un efecto destacado los factores del entorno socioeconómico, los relacionados con el propio diputado, así como los que tienen que ver con el partido al que él pertenece.

Para contestar estos interrogantes se ha dividido el artículo en cinco secciones. En la primera se revisan brevemente algunas de las investigaciones más destacadas sobre modos de representación y se justifica la intención de indagar en el nivel distrital. En la segunda se detallan los casos y datos del análisis empírico, aunque cabe aclarar que el grueso de la evidencia empírica proviene de una encuesta a asesores parlamentarios de los principales partidos bolivianos y

1 Este trabajo fue realizado con el apoyo del proyecto "Los vínculos entre electores y partidos: la actividad distrital de los diputados de Chile, Perú y Bolivia" (CSO2011-24344) financiado por el Ministerio de Ciencia e Innovación de Espańa. 
chilenos. En la tercera se comparan las preferencias de los diputados bolivianos y chilenos respecto a los intereses de representación en su actividad distrital. La cuarta se ocupa del efecto de las variables explicativas relativas al entorno, al diputado y al partido que contribuyen a comprender las variaciones observadas en relación con tales preferencias. Y, finalmente, se presentan las conclusiones generales.

\section{Los modos de representación política}

La reflexión sobre el modo en que los parlamentarios deben ejercer su labor de representación, así como la naturaleza de la relación que deben mantener con los representados constituye uno de los debates clásicos en la literatura sobre la representación política. Ya en los inicios del sistema de gobierno representativo se evidenciaron dos concepciones contrapuestas que han perdurado hasta la actualidad: la controversia mandato versus independencia (Pitkin, 1967). De acuerdo con la primera concepción, el representante debe hacer lo que los electores quieren y, por tanto, debe ajustarse a sus instrucciones (o mandatos). En cambio, de acuerdo con la segunda concepción, el representante debe sentirse libre para seguir su propio criterio en la consecución del bienestar de sus electores. Esta contraposición es el punto de arranque de una discusión más amplia de la que, a continuación, se revisan algunas de las aristas más relevantes en relación con nuestras preguntas de investigación.

\section{La literatura sobre los modos de representación}

Por "modos de representación" nos referimos a las formas en que los parlamentarios desempeñan su actividad de representación y establecen relaciones con los votantes (Andeweg \& Thomassen, 2005). Son diversas las cuestiones abordadas en la literatura al respecto, pero sobresalen tres: a quién representan los parlamentarios, cómo estos realizan la representación, y hasta qué punto los parlamentarios son sensibles a las preferencias de los votantes (Weßels, 2007; Bengtsson \& Wass, 2010). La primera de estas cuestiones se refiere al foco de la representación, es decir, a quién representa el parlamentario en su actividad. En general, la literatura ha destacado tres tipos de intereses que pueden defender los representantes: los territoriales, que van desde los intereses de todo el país a los de regiones y distritos electorales; los funcionales, vinculados a intereses de diferentes grupos y sectores sociales (étnicos, religiosos, económicos, etc.), y los de los partidos a los que pertenecen los representantes (Eulau 
et al., 1959; Brack et al., 2012). Este artículo se sitúa dentro de la primera línea investigativa y centra su atención en dos tipos de intereses a los que tratan de atender los parlamentarios en su actividad de representación: los de su distrito electoral y los de su partido.

La segunda cuestión concierne al estilo de representación, esto es, a la forma en que los parlamentarios representan a sus respectivos principales y al nivel de autonomía de que disfrutan en relación con ellos. ${ }^{2}$ Dos casos muy ilustrativos de estilos de representación son los mencionados de mandato e independencia —o free agency - (Eulau et al., 1959). La tercera cuestión del debate sobre modos de representación se refiere a la congruencia entre las preferencias de los parlamentarios y la de los votantes. Algunos autores han sostenido que la representación basada en el modelo de gobierno de partido responsable es la que más asegura dicha congruencia. Según este modelo, los partidos presentan en las elecciones diferentes políticas que pretenden impulsar desde el gobierno, a partir de las cuales los ciudadanos orientan su voto (Adams, 2001). Otros autores han formulado severas críticas a este modelo; entre otras, se ha destacado que constituye una visión idealizada del gobierno representativo, sobre todo, en cuanto al cumplimiento de las promesas electorales (Brack et al., 2012).

Una inquietud extendida entre los que se han ocupado de los modos de representación es la de conceptualizar y clasificar su objeto de investigación, prestando una particular atención a la clase de intereses defendidos por los parlamentarios, es decir, al foco de la representación. Así, en la teoría política normativa se pueden apreciar diferentes clasificaciones de modos de representación que tienen en cuenta tales intereses; baste mencionar tres de las más conocidas. Una es la distinción entre modelos republicanos y liberales o pluralistas de representación (Offe \& Preuss, 1990; Rehfeld, 2009). Para los republicanos la finalidad de la representación es la consecución del interés general de toda la comunidad, mientras que para los liberales o pluralistas se trata de la garantía de los intereses particulares que existen dentro de la comunidad. Otra clasificación es la de Pitkin (1967), la cual se centra en los estilos de representación, pero toma en cuenta la cuestión de los intereses, y que establece una diferencia entre representación descriptiva, por la cual se concibe al representante no tanto como alguien que actúa por otros, sino que los sustituye, y representación sus-

2 Se utiliza principal en el sentido atribuido en la teoría de la agencia (Mitnick, 2013). Esta teoría, aplicada al ámbito de la representación, concibe al representante como un agente, que actúa por delegación de otro - el principal - y que trata de satisfacer las preferencias de este último. Esta situación sitúa al representante ante un dilema: cumplir con los términos del contrato de representación por el que fue designado o perseguir sus propios intereses (Fiorina, 1981). 
tantiva, que implica que el representante puede actuar libremente, de acuerdo con lo que él considera que es lo mejor para el interés de los votantes. ${ }^{3}$ Por último, Mansbridge $(2003,2011)$ ha revisado la dicotomía clásica de mandato versus independencia y ha elaborado otra tipología de modelos de representación que, entre otros criterios, considera el foco de la representación; en concreto, plantea cuatro formas de representación: basada en promesas, anticipativa, giroscópica, y suplente. ${ }^{4}$

Pero ha sido en la ciencia política empírica donde se ha concedido mayor relevancia al foco de representación como criterio de clasificación de los modos de representación y donde se delimitan, de forma más precisa, la tipología de intereses que pueden defender los parlamentarios. La más conocida es la de Eulau et al. (1959), la cual perfila diferentes roles de legisladores en función de dos dimensiones: el foco y el estilo de la representación. Aunque señalan que la cuestión del foco se refiere a distintas clases de intereses funcionales y territoriales priorizados por los parlamentarios, su estudio empírico se limita a los intereses de representar a cierto territorio (distrito electoral, Estado y una combinación de ambos). Junto a esto, diferencian tres estilos de representantes: los trustees, que se ajustan al ideal de representante de Burke, esto es, aquel que está guiado únicamente por su propio juicio; los delegados, que desarrollan una representación por mandato (siguen las instrucciones de los votantes); y los políticos, que se comportan como delegados o como trustees en función de las circunstancias. A pesar de la relevancia de esta clasificación en la investigación sobre modos de representación (Thomassen, 1994; Méndez-Lago \& Martínez, 2002; García \& Marenghi, 2008), ha sido objeto de crítica reciente. Entre otras cuestiones, se ha señalado la ambigüedad de su aplicación empírica, así como su limitación al omitir la influencia que ejercen los partidos sobre los representantes (Andeweg \& Thomassen, 2005). Ante estas insuficiencias, Andeweg \& Thomassen (2005) han elaborado una nueva tipología, que identifica cuatro modos de representación (autorización, delegación, responsiveness y accountability), y con la que han examinado el caso holandés.

3 En el trabajo de Pitkin (1967) pueden encontrarse otras concepciones de representación: como autorización, como responsabilidad y simbólica. Véase también Martínez (2004).

4 La primera forma de representación corresponde al modelo tradicional: las promesas de gobierno de los partidos en las campańas electorales orientan su acción representativa; la representación anticipativa está vinculada a la idea del voto retrospectivo: los representantes se enfocan en lo que creen que los votantes evaluarán en las futuras elecciones y no en lo que prometieron en el pasado; en la representación giroscópica, los representantes están internamente motivados y actúan sobre la base de unos principios y objetivos fijos; y la representación suplente es aquella que no tiene relación electoral directa: el parlamentario representa a votantes de distritos diferentes al suyo (Mansbridge, 2003, 2011).

M. Barreda Díaz, L. M. Ruiz Rodríguez | Modos de representación en los distritos: ¿̨diputados al servicio de los partidos o de los electores? Los casos de Chile y Bolivia | Perfiles Latinoamericanos, 25(50) | Flacso México 
Así las cosas, frente al amplio interés teórico que ha suscitado el tema de los modos de representación política, no puede decirse lo mismo sobre las aproximaciones empíricas. Desde el análisis pionero de Eulau et al. (1959), apenas un número reducido ha abordado esta temática y, además, lo han hecho desde una misma perspectiva: atendiendo a las visiones de los propios parlamentarios (Converse \& Pierce, 1979; Andeweg \& Thomassen, 2005; Brack et al., 2012; Deschouwer \& Depauw, 2014). Frente a esta tendencia general, algunas investigaciones recientes han dirigido la atención hacia los ciudadanos, con el propósito de examinar sus preferencias sobre los modos de representación (Méndez-Lago \& Martínez, 2002; Carman, 2007; Barker \& Carman, 2010; Bengtsson $\&$ Wass, 2010). La comparación entre toda esta literatura resulta bastante compleja, dada la diversidad de marcos conceptuales y metodologías utilizadas (Bengtsson \& Wass, 2010). Aun así, todos convergen en enfatizar la notable variedad de preferencias de modos de representación existentes, lo que se aprecia, por ejemplo, al comparar las actitudes de parlamentarios de distintos partidos, distritos y países (Brack et al., 2012).

En lo que se refiere a los determinantes de las preferencias de los parlamentarios por un modo u otro de representación, la investigación es todavía bastante limitada. Pero incluso así, se constata una conclusión general: la naturaleza multicausal de tales preferencias. En particular, aquellos que han analizado los modos de representación mediante encuestas a parlamentarios han mostrado la incidencia de variables relativas a sus propias características, a su partido y al entorno institucional (Brack et al., 2012). Por su parte, la investigación basada en encuestas a ciudadanos ha resaltado el impacto de las variables sociodemográficas y, en menor medida, de variables relacionadas con la cultura política como la eficacia política y la identificación partidista (Carman, 2007; Bengtsson \& Wass, 2010). Algunos trabajos recientes han dado un paso más allá en el análisis de las preferencias ciudadanas sobre modos de representación al examinar sus efectos políticos. En concreto, Barker \& Carman (2010) han comprobado la influencia de dichas preferencias en la decisión de voto de los ciudadanos estadounidenses (tanto en elecciones primarias como en las presidenciales).

Dentro de la literatura sobre modos de representación política, América Latina ha padecido de atención. Entre lo poco hallado se encuentra la investigación comparada de García Montero \& Marenghi (2008) de los roles de los parlamentarios latinoamericanos, y algunos análisis que han elaborado tipologías adaptadas a la región sobre temas cercanos a los modos de representación. Así podemos catalogar la clasificación de Contreras (2012) sobre la intermediación de cargos políticos, y la clasificación de candidatos de Siavelis \& Morgenstern (2008), la cual distingue entre empresarios políticos, leales al partido, servidores del distrito y delegados de grupo. 
La tarea de representación de los parlamentarios discurre fundamentalmente en tres ámbitos: la cámara legislativa, el partido y el distrito electoral (Davidson et al., 2014). El objeto de investigación de la actividad de representación y, en particular, de los roles que desempeñan los parlamentarios ha sido la esfera legislativa. No obstante, algunos países, en especial Estados Unidos, cuentan con una tradición de investigación sobre dicha labor en los distritos electorales. En este sentido destacan en primer lugar Mayhew (1974), Fiorina (1977) y, sobre todo, Fenno (1978), autor que se convirtió en la piedra de toque de la investigación sobre la actividad de los parlamentarios en los distritos. Fenno realiza un análisis cualitativo, casi etnográfico, de los estilos y recursos utilizados por los congresistas estadounidenses en su actividad permanente en la circunscripción (en su home sytle), ${ }^{5}$ así como los condicionantes de esta actividad.

Tras cuatro décadas de investigación empírica sobre distritos y representación en Estados Unidos, una de las principales conclusiones que se constata es la simultaneidad entre un componente colectivista y otro individualista en el modo de representación de los legisladores (Ansolabehere $\&$ Jones, 2011). Por un lado, los parlamentarios operan como integrantes de un partido y, sobre la base de su identidad partidista, buscan el apoyo del electorado. Pero, por otro, cultivan vínculos personales con sus electores, a veces a expensas del propio partido.

En contraste con el conocimiento generado a propósito del caso norteamericano, en América Latina se sabe muy poco de lo que sucede en el ámbito distrital de la representación. Este déficit resulta llamativo, entre otras razones, porque la regulación parlamentaria de muchos países de la región estipula las condiciones para que los legisladores puedan dedicar parte del tiempo a la presencia y actividad en sus distritos. ${ }^{6}$ Entre las contadas aproximaciones al ámbito distrital, destaca Valenzuela (1977), autor que retrata a los diputados chilenos como political brokers e insertos en redes de intermediación (junto con alcaldes, partidos y electores). En la misma línea, Díaz et al. (2006) y Contreras (2012) han descubierto que los diputados chilenos forman parte, en sus distritos, de

5 Fenno (1978) utiliza este término para referirse a la actividad en los distritos, frente al de hill style, relativo al interior del Congreso, que alude al Capitol Hill de Washington, D. C. (sede del Legislativo norteamericano).

6 Basten como ejemplo los dos casos tratados aquí. En Chile se establece que el trabajo legislativo de cada mes se distribuye en tres semanas legislativas — de trabajo en comisiones y/o Pleno- y una semana distrital, en la que los diputados viajan a sus distritos. En Bolivia se fija que una cuarta parte de las sesiones en la Cámara de Diputados pueden ser con la asistencia de los diputados suplentes. Se entiende que esa semana de ausencia es para acudir a los distritos.

M. Barreda Díaz, L. M. Ruiz Rodríguez | Modos de representación en los distritos: ¿̨diputados al servicio de los partidos o de los electores? Los casos de Chile y Bolivia | Perfiles Latinoamericanos, 25(50) | FLAcso México 
redes más amplias de intermediación, de mayor permanencia (no solo en periodos electorales) y con niveles más elevados de personalismo. Asimismo, los análisis sobre clientelismo se han aproximado al ámbito distrital y han aportado información sobre la relación entre candidatos y potenciales votantes, sobre todo durante el periodo electoral (por ejemplo, Brusco et al., 2004; Amaral \& Stokes, 2005; Szwarcberg, 2013). Asimismo en Luna (2014), quien se ocupa de las estrategias de los partidos políticos, se aprecian algunas claves de la actividad de estos últimos en los distritos de Chile y Uruguay.

En este contexto, el presente artículo contribuye al análisis de la representación en América Latina, concentrándose en el nivel distrital. El propósito es examinar qué rol predominante desempeñan los parlamentarios en su actividad distrital en tiempos no electorales. Los periodos ordinarios permiten hacer un balance sobre los mecanismos de representación que emplea un diputado. Por el contrario, las entrevistas en el periodo extraordinario que supone una campaña electoral dan pauta para capturar las estrategias de captación de votos, pero no los mecanismos de representación. ${ }^{7}$ Para este análisis se toma en cuenta la distinción planteada para el caso norteamericano entre una orientación colectiva (o partidista) y una individual, que atiende a los deseos del electorado. Con esta aproximación se proporciona una comprensión más profunda de los procesos de representación política al acercarnos al modo en que los diputados se vinculan con sus electores, así como al posible papel de los partidos como entes mediadores en esta relación de representación. Cabría esperar que los partidos más institucionalizados tuvieran más capacidad para condicionar el modo de representación de sus diputados en los distritos. Pero, como se verá más adelante, en esta ecuación influyen otros factores del entorno y del propio diputado que hacen necesaria una argumentación más compleja en torno al triángulo de partidos, diputados y distritos.

\section{Selección de casos y datos}

Este artículo analiza los modos de representación de los diputados de Bolivia y Chile en su actividad distrital. Los sistemas políticos de estos dos países han experimentado procesos históricos distintos y también se diferencian en su diseño institucional en aspectos como su sistema electoral, su grado de descen-

Stokes (2013) aborda las estrategias clientelares de diputados y partidos en periodos electorales. Mientras que Szwarcberg (2012) aporta evidencia empírica sobre el modo en que se construye la relación entre el partido y sus brokers (los diputados) durante las campańas electorales. Ambos autores ejemplifican un objeto de estudio relacionado con nuestra temática, pero diferente en términos sustantivos. 
tralización — por citar dos variables—, así como en el tema específico que nos ocupa, esto es, las características de la representación política. Bolivia es uno de los países latinoamericanos que ha experimentado más acusadamente una crisis de representación (con aumento de la desafección, volatilidad, fluidez y fragmentación), que ha desembocado en una mutación radical del sistema de partidos y del modelo de gobernabilidad establecido. Algunas señales de ello fueron la irrupción de nuevos actores políticos, la concentración del poder en el MAS y la desaparición de la dinámica de pactos y gobiernos de coalición entre partidos (Mainwaring et al., 2006; Domingo, 2006). En el caso de Chile, aunque ha visto aumentar el nivel de desafección política y atraviesa una desestructuración programática y desinstitucionalización (Luna \& Mardones, 2010), sigue contando con uno de los sistemas de partidos más institucionalizados de la región y con partidos de procedimientos rutinizados y estructuración programática, aunque ello se combina con crecientes críticas relacionadas con la limitada renovación de las élites políticas, que repercuten negativamente en la calidad de la representación política.

En este contexto de diferencias entre países, aquí se realiza una comparación entre sus partidos políticos. Con los hallazgos se confirmará si se producen variaciones en el foco de la representación como resultado de factores relativos a los diputados, y a la pertenencia a organizaciones partidistas con recursos distintos en lo que a institucionalización y estructuración se refiere. $\mathrm{Al}$ mismo tiempo, los contrastes entre Chile y Bolivia enriquecen este análisis, pues hacen posible la comparación de sus diferencias en el foco de la representación como resultado de factores institucionales y contextuales. No obstante, y pese a la utilidad del procedimiento, las conclusiones sobre el impacto de las variables se basan en un análisis estadístico que permite controlar la variación entre casos y países, al medir el efecto causal de cada una de las variables independientes sobre el tipo de representación que ejercen los diputados.

$\mathrm{El}$ análisis de los modos de representación se sustenta sobre todo en una encuesta realizada entre asesores de diputados durante los meses de febrero a abril de 2013. Además de la disposición de los encuestados, otras dos razones le dan fiabilidad a esta fuente de información. Por una parte, los asesores conocen directamente la labor de representación del diputado y les llevan su agenda, lo que los convierte en observadores privilegiados. ${ }^{8}$ Por otra parte, los asesores disponen de mayor distancia para evaluar la relación del diputado con el distrito y con el partido, lo que disminuye su predisposición al sesgo en las respuestas. Para determinar el listado de asesores que serían encuestados se siguieron dos

8 Esta encuesta a asesores constituye una base reconocida que ha sido utilizada en otros trabajos como los de Barreda \& Ruiz (2014) y Corrales, Sánchez \& Rivas (2016). 
pasos: 1) fijar una muestra de diputados que combinase la representación de los principales partidos junto con la diversidad de distritos electorales de cada país, 2) contactar después con la oficina del diputado en el Congreso y/o en su distrito. En total, se entrevistó a asesores de diputados de ocho partidos, elegidos en 32 distritos electorales: Movimiento al Socialismo (MAS), Partido por BoliviaConvergencia (ррв-Convergencia), Unidad Nacional (UN) y Alianza Social (As), en Bolivia; Renovación Nacional (RN), Partido por la Democracia (PPD), Partido Demócrata Cristiano (PDC) y Unión Demócrata Independiente (UDI), en Chile.? Esta distribución de casos reúne circunscripciones variadas en rasgos demográficos e institucionales de los tres países. Además de la encuesta de asesores, se ha recurrido a datos secundarios — como resultados electorales y de desarrollo socioeconómico — para el análisis explicativo de la actividad distrital.

\section{El foco de representación de los diputados chilenos y bolivianos en su actividad distrital}

Como se ha dicho, la literatura ha puesto de relieve maneras muy diversas de conceptualizar y de analizar empíricamente los modos de representación. Hay quienes centran la atención en el estilo y otros en el foco, mientras que unos más abordan ambas cuestiones, de forma combinada o separada (Bengtsson $\&$ Wass, 2010). El presente artículo se incluye en las investigaciones sobre el foco de representación y se ocupa, en concreto, de examinar qué intereses priorizan los diputados en su actividad distrital: los intereses de su partido o los de los ciudadanos. En otras palabras, se trata de ver si los diputados tienden a comportarse como servidores de partidos o de los ciudadanos. Nuestra encuesta a asesores de diputados incluye dos preguntas que dan pie para examinar estas relaciones de agencia. Una de ellas se enfoca a valorar con quién se vincula más el diputado cuando se encuentra en su circunscripción: ¿con la sociedad civil o con su partido político? La otra pregunta se refiere a quién influye más en los procesos de toma de decisiones: la sociedad civil o el partido. En ambos casos se utiliza una escala de 1 (sociedad civil) a 10 (partido). A sabiendas de que con frecuencia la estrategia de los diputados es doble, pues combina la atención al partido y al distrito, el objetivo es capturar qué aspecto pesa más en el conjunto de su labor.

9 El tamaño limitado del universo de estudio (asesores de diputados) unido a las dificultades habituales de la no respuesta a cuestionarios explican que la $n$ de la encuesta sea reducida (74). Esta limitación cuantitativa se ha tratado de suplir — "cualitativamente" — con una selección lo más rigurosa posible de los asesores de los diputados, de manera que reflejen la diversidad partidista y sociodemográfica de cada país. Para más detalle de la muestra, véase el anexo. 
Como se aprecia en el cuadro 1, el promedio de los diputados chilenos y bolivianos se inclina por priorizar los intereses ciudadanos de su distrito frente a los de sus partidos. ${ }^{10}$ Resultados similares, a partir de otros indicadores y datos, obtuvieron García \& Marenghi (2008) sobre los parlamentarios latinoamericanos. Si bien la preeminencia de los intereses ciudadanos es generalizada, hay una diferencia significativa entre los diputados bolivianos y los chilenos: los primeros anteponen en mayor grado la representación de los intereses partidistas.

$\mathrm{El}$ hecho de que los diputados del MAS estén más predispuestos a representar los intereses partidistas en el ámbito distrital en comparación con los partidos chilenos puede ser, a primera vista, sorprendente. El sistema de partidos de Bolivia está menos institucionalizado que el chileno, el cual tiene partidos más antiguos y estructurados. Por esto se esperaría que los partidos chilenos tuvieran más capacidad para condicionar la actividad de los diputados en los distritos. Al mismo tiempo, dado que el MAS es un partido joven, con bajo nivel de institucionalización y creado como "instrumento político" de los movimientos sociales y organizaciones sindicales del mundo rural (Do Alto \& Stefanoni, 2009), cabría esperar, a priori, una representación más focalizada en los intereses sociales del distrito. Sin embargo, los diputados bolivianos y, en especial los del MAS, privilegian el servir a los partidos mientras que los chilenos están más orientados al distrito.

Cuadro 1. Foco de representación (sociedad civil-partido) de los diputados chilenos y bolivianos en su actividad distrital

\begin{tabular}{lcccccc}
\hline & $M A S$ & $P P B-C N$ & $D C$ & $P P D$ & $R N$ & Total \\
\hline $\begin{array}{l}\text { Con quién se relaciona más el diputado } \\
\text { en la circunscripción: }\end{array}$ & & & & & & \\
sociedad civil (1)-partido (10) & & & & & & \\
Media & 4.4 & 3.5 & 2.8 & 2.2 & 3.3 & 3.6 \\
Desviación típica & 2.2 & 2.2 & 0.8 & 1.3 & 0.7 & 2 \\
$\mathrm{~N}$ & $(27)$ & $(15)$ & $(9)$ & $(9)$ & $(9)$ & $(69)$ \\
Quién le influye más en los procesos & & & & & & \\
de toma de decisiones: & & & & & & \\
sociedad civil (1)-partido (10) & & & & & & \\
Media & 4.9 & 4 & 2.9 & 2 & 3 & 3.8 \\
Desviación típica & 2.3 & 2.2 & 0.9 & 0.9 & 0.7 & 2.1 \\
$\mathrm{~N}$ & $(27)$ & $(15)$ & $(9)$ & $(9)$ & $(9)$ & $(69)$ \\
\hline
\end{tabular}

Fuente: Encuesta de Asesores de Diputados (2013).

Para comprender esta aparente paradoja conviene revisar el impacto de los aspectos del entorno junto con los del partido y los del diputado. En cuanto al entorno, aunque hay diferencias entre los partidos de un mismo país (por

10 Del análisis comparativo por partidos, a tres de estos se les ha excluido. Se trata de AS y un, que tienen una representación muy minoritaria en el Congreso de Bolivia, y de uDI, para el que se dispone de un número reducido de asesores encuestados (véase el anexo).

M. Barreda Díaz, L. M. Ruiz Rodríguez | Modos de representación en los distritos: ¿diputados al servicio de los partidos o de los electores? Los casos de Chile y Bolivia | Perfiles Latinoamericanos, 25(50) | FLACso México 
ejemplo, la orientación propartido es más marcada en el MAS que en el PPB-CN), son menos acusadas que las que se aprecian entre diputados de uno y otro país. Esto se constata también cuando se examina la variación en el interior de los partidos. Así, los dos partidos bolivianos presentan desviaciones típicas más elevadas y, por tanto, evidencian una mayor disparidad interna relativa a la priorización de los intereses partidistas o ciudadanos. Todo ello sugiere que los factores del entorno social e institucional propios de cada país son relevantes y que afectan a sus respectivos sistemas de partidos.

En lo que se refiere al impacto de las variables partidistas, cabe destacar el cambio de preferencias de los parlamentarios acerca del foco de representación, como resultado del nuevo marco de incentivos que ha transformado al MAS en un partido de gobierno. En especial, la fuerte concentración del poder en manos del líder (Evo Morales), que afecta al control sobre los parlamentarios, unido a la profesionalización de los representantes y las posibilidades de acceso a puestos públicos (las denominadas "pegas") han incentivado comportamientos alineados con los intereses del partido, por encima de los sociales o distritales. ${ }^{11}$

En este contexto, el apartado siguiente comprueba con métodos estadísticos si los factores partidistas del entorno y del diputado tienen pesos variables en la preferencia por un determinado foco de representación.

\section{Una exploración de los determinantes del foco de la representación}

Se acaba de ver que los diputados bolivianos y chilenos coinciden en focalizar la representación en los intereses ciudadanos del distrito, pero con notables variaciones según el país y el partido; determinar qué las causa es un asunto complejo, ya que exige considerar numerosos elementos potencialmente explicativos. La literatura empírica que ha tratado de comprender los modos de representación señala factores muy diversos. En particular, se ha resaltado la incidencia del perfil de los representantes, como la experiencia política y las responsabilidades dentro del partido; el tipo de partido y su organización, por ejemplo, el nivel de centralización y de democracia interna; y el contexto institucional en que operan los representantes, sobre todo el sistema electoral (Brack et al. 2012; García Montero \& Marenghi, 2007). En un sentido más general, muchas de las variables con que se ha explicado la representación en América Latina se podrían aplicar a los determinantes de los modos de representación,

11 El gobierno de Evo Morales utiliza la distribución de pegas entre los miembros del MAS (incluidos los diputados) como incentivos selectivos para lograr la unidad en el partido y que se apoye al ejecutivo (Do Alto \& Stefanoni, 2009). Sobre los cambios en la distribución interna de poder en el MAS, véase Mayorga (2008). 
de las que algunas de las principales son: las características de los legisladores, como su poder en el partido (Siavelis \& Morgenstern, 2008; Luna, 2010) y la reelección (Carroll \& Shugart, 2007); aspectos partidistas como la ideología y el rendimiento electoral (Alcántara, 2004), la financiación (Luna, 2010) y la estructuración programática (Ruiz Rodríguez, 2007), y las características socioeconómicas de los distritos electorales (Luna, 2010).

Dada la diversidad de variables potencialmente explicativas y la escasez de investigación empírica sobre ello, se plantea aquí un trabajo exploratorio para mostrar algunas claves de las distintas preferencias de los diputados por determinados focos de representación. El modelo analítico utilizado se expone a continuación.

\section{Modelo analítico}

La exploración de los determinantes de los focos de representación de los diputados en el nivel distrital se sustenta en un modelo analítico que incluye los aportes de la literatura sobre el tema. Como variable dependiente se consideran las dos preguntas de nuestra encuesta a asesores parlamentarios, las cuales miden la priorización de intereses de representación de los diputados en los distritos: "con quién se relaciona más: con la sociedad civil o con el partido" y "quién le influye más: la sociedad civil o el partido". En consecuencia, nuestra variable dependiente captura la predisposición promedio de los diputados para atender un tipo de intereses u otros. Presuponemos que en su tarea de representación ellos combinan una estrategia de atención al partido y al distrito, pero en este artículo, con la evidencia empírica disponible, solo podemos analizar qué estrategia predomina y qué variables lo explican. Las dos preguntas son variables ordinales, lo que limitaría su uso en un análisis de regresión lineal. Sin embargo, si se tiene en cuenta el alto número de valores de cada una (de 1 a 10) y que las distribuciones de estas variables se aproximan a las de una curva normal, ${ }^{12}$ se las puede considerar como variables cuantitativas discretas y, con esto, susceptibles de aplicarse a este tipo de análisis estadístico.

En cuanto a las variables independientes, se han seleccionado con dos criterios: 1) que se trate de un número reducido de variables, dada la limitada cantidad de observaciones de las dos encuestas; ${ }^{13}$ y 2) que estén conectadas con

12 El estadístico de asimetría de la variable 1 (“con quién se relaciona más”) es de 0.66 y el de la variable 2 ("quién le influye más") es de 0.42 . Por su parte, los estadísticos de curtosis son de 0.49 y - 0.43 , respectivamente. Estos resultados indican que las distribuciones de las variables se aproximan a la normal.

13 Hay que ańadir que algunas preguntas de interés de la "Encuesta de Asesores a Diputados" (por ejemplo, relativas a la financiación o el grado de centralización del partido) obtuvieron un alto número de 
explicaciones teóricas consistentes. Así, se han seleccionado siete variables sobre las tres clases de explicaciones de modos de representación destacadas en la literatura (relativas a los diputados, a los partidos y al entorno en que operan). Ello permitirá apreciar el peso de unos y otros grupos de factores en la gestación de diputados con perfil pro partido o pro distrito. A continuación se presentan y justifican dichas variables.

Del grupo de variables que corresponden a las características de los diputados, se han seleccionado tres. La primera es la carrera política del diputado. De acuerdo con algunos autores, los parlamentarios con mayor experiencia política se inclinan por una representación focalizada en los intereses del distrito, por encima de los de sus partidos y sus votantes (Brack et al., 2012), así como por un estilo independiente de representación (García \& Marenghi, 2008). La segunda variable es la ubicación en el eje izquierda-derecha. Tras revisar diversas encuestas nacionales a parlamentarios, Gauja (2013) concluye que el foco de representación varía considerablemente según la posición en este eje. Los diputados de izquierda, en comparación con los de derecha, tienden a priorizar los intereses del partido. La tercera variable es el uso de Internet por parte del diputado, habida cuenta de los beneficios que supone en el ejercicio de representación. Por ejemplo, se ha destacado que usar la red abarata los costes de la comunicación política, estrecha los vínculos entre representantes y representados, y que de estos beneficios se pueden aprovechar todos los actores políticos, con independencia de sus recursos estratégicos (Gibson et al., 2003; Padró-Solanet \& Cardenal, 2009). De acuerdo con esto, el diputado que utiliza Internet puede tener mayor autonomía respecto de su partido cuando organiza y desarrolla la actividad distrital.

Asimismo, se han seleccionado dos variables que involucran a los partidos de los diputados para medir su impacto en la representación. Una de ellas es si el partido se ubica en el gobierno o en la oposición. Como se indicó al tratar el caso del MAS, pertenecer a un partido en el gobierno favorece que el foco de la representación sean los intereses partidistas y no los del distrito; entre otras razones, porque los partidos en el gobierno pueden brindar a sus miembros incentivos selectivos que facilitan la unidad y la disciplina interna (Mainwaring, 1999). La otra variable se refiere a un aspecto de la organización de los partidos de particular interés para el análisis de la representación en la esfera distrital: la presencia de una sede del partido en la circunscripción del diputado. Si esto sucediera, es probable que se favoreciera una actividad de representación más alineada con las preferencias y orientaciones del partido.

NS/NC, por lo que se han excluido del modelo. Son cuestiones que, o no conocen algunos asesores con precisión, o prefieren no contestar. 
Finalmente, se han valorado dos variables del entorno institucional y socioeconómico en el que operan los representantes. La primera se refiere a un elemento del sistema electoral: la magnitud de los distritos. La tesis comúnmente aceptada es que los distritos uninominales incentivan, en contraste con los plurinominales, que la lealtad del representante sea con los votantes del distrito y no con su partido (Cox, 2004; Carroll \& Shugart, 2007). En este sentido, cabría esperar que los diputados de circunscripciones pequeñas consideren a la ciudadanía de su distrito como su principal. La última variable independiente es el nivel de desarrollo de cada circunscripción. Estudios recientes han destacado que las características socioeconómicas de los distritos afectan al desempeño de la representación; de este modo, los de bajo nivel de desarrollo favorecen las relaciones de intercambio político personal y clientelar (Luna, 2010). De acuerdo con esto, a mayor nivel de desarrollo habrá mayor tendencia a una representación al servicio del partido.

El cuadro 2 detalla los indicadores utilizados para las siete variables independientes presentadas, y las relaciones de causalidad que se esperan. Se ha agregado la variable pais por las diferencias entre diputados chilenos y bolivianos ya comentadas respecto al foco de la representación.

Cuadro 2. Variables independientes del modelo

\begin{tabular}{|c|c|c|}
\hline Variables & Indicadores & Relaciones de causalidad esperadas \\
\hline & & Relativas a diputados \\
\hline Carrera política & $\begin{array}{l}\text { Primera legislatura (o no) } \\
\text { del diputado }\end{array}$ & $\begin{array}{l}\text { Los diputados con experiencia en ese cargo tienden a priorizar los } \\
\text { intereses de sus distritos, por encima de los de sus partidos }\end{array}$ \\
\hline Ideología & $\begin{array}{l}\text { Posición del diputado en } \\
\text { eje izquierda-derecha }\end{array}$ & $\begin{array}{l}\text { Los diputados ubicados en la izquierda favorecen más los intereses } \\
\text { de sus partidos }\end{array}$ \\
\hline Uso de Internet & $\begin{array}{l}\text { Valoración del uso de } \\
\text { Internet en actividad } \\
\text { distrital del diputado }\end{array}$ & $\begin{array}{l}\text { Cuanto mayor es la importancia atribuida al uso de Internet, menor } \\
\text { es la tendencia a priorizar la defensa de los intereses de los partidos }\end{array}$ \\
\hline & & Relativas a partidos \\
\hline $\begin{array}{l}\text { Gobierno- } \\
\text { oposición }\end{array}$ & $\begin{array}{l}\text { Ubicación del partido del } \\
\text { diputado: en gobierno u } \\
\text { oposición }\end{array}$ & $\begin{array}{l}\text { La pertenencia a un partido en el gobierno favorece que el foco de la } \\
\text { representación sea defender los intereses del partido }\end{array}$ \\
\hline $\begin{array}{l}\text { Presencia del } \\
\text { partido en distrito }\end{array}$ & $\begin{array}{l}\text { El partido tiene o no } \\
\text { sede en el distrito }\end{array}$ & $\begin{array}{l}\text { Que haya una sede en el distrito favorece una actividad de represen- } \\
\text { tación más alineada con las preferencias y orientaciones del partido }\end{array}$ \\
\hline & & Relativas a entorno \\
\hline $\begin{array}{l}\text { Magnitud de } \\
\text { distritos }\end{array}$ & Magnitud de los distritos & $\begin{array}{l}\text { En los distritos de tamaño pequeño hay mayor inclinación a desa- } \\
\text { rrollar una representación orientada a satisfacer las preferencias del } \\
\text { distrito }\end{array}$ \\
\hline $\begin{array}{l}\text { Desarrollo socio- } \\
\text { económico de los } \\
\text { distritos }\end{array}$ & Nivel de pobreza & $\begin{array}{l}\text { A mayor nivel de desarrollo y menor pobreza, mayor tendencia a } \\
\text { representar al servicio del partido }\end{array}$ \\
\hline País (Chile-Bolivia) & & \\
\hline
\end{tabular}

Fuentes: Los indicadores de las variables de carrera política, ideología, uso de Internet y presencia del partido en distrito proceden de la Encuesta a Asesores de Diputados (2013); los indicadores de ubicación en gobierno-oposición y magnitudes de distritos se han elaborado con información de las Cámaras de Diputados de ambos países; y el indicador de nivel de pobreza, a partir de datos del INE de Bolivia y el SINIM de Chile.

M. Barreda Díaz, L. M. Ruiz Rodríguez | Modos de representación en los distritos: ¿̨diputados al servicio de los partidos o de los electores? Los casos de Chile y Bolivia | Perfiles Latinoamericanos, 25(50) | FLAcso México 
Con el modelo elaborado se ha realizado un análisis de regresión lineal (cuadro 3). Las variables con mayor capacidad explicativa de los focos de representación son el país del diputado, la magnitud del distrito y el nivel de pobreza de este. Las tres variables son estadísticamente significativas en los dos modelos. De las variables independientes restantes, solo la ubicación en el eje izquierdaderecha no presenta relación significativa en los dos modelos.

La variable más explicativa de todas las incluidas (con el coeficiente tipificado, beta, más elevado) es la de país. Ello corrobora las diferencias ya indicadas entre los diputados chilenos y bolivianos: los primeros priorizan los intereses de los partidos en su actividad distrital más que los segundos. De manera que los elementos específicos del contexto del país influyen en los modos de representación. En particular, un aspecto del sistema electoral desempeña un papel clave: la magnitud de las circunscripciones. En Chile estas tienen el mismo tamaño (2), mientras que en Bolivia las hay uninominales y plurinominales. La dimensión de los distritos no solo genera variaciones entre los diputados de los dos países, sino también entre los diputados bolivianos, ${ }^{14}$ las cuales van en el mismo sentido: a mayor tamaño de los distritos aumentará la focalización en intereses partidistas. Otra característica de los distritos electorales que afecta al modo de representación es el nivel de desarrollo. Los diputados de distritos pobres priorizan en menor medida los intereses del partido en su labor. Como se señalaba, estos diputados tienen más incentivos para establecer relaciones de intercambio personal y clientelar que los diputados de circunscripciones prósperas.

Por lo que se refiere a las demás variables independientes, tres de ellas reportan una relación significativa con el modelo 2 y su incidencia se ajusta a lo esperado: la limitada carrera política del diputado, que el partido se encuentre en el distrito y la condición de partido en el gobierno favorecen una representación alineada a las preferencias del partido. Esto último reafirma la explicación ya expuesta sobre el MAs: su posición en el gobierno brinda a sus diputados incentivos selectivos que inducen a priorizar los intereses del partido por encima de los del distrito. La última variable que resulta explicativa (en el modelo 1) es el uso de Internet. En concordancia con los argumentos ya dichos, la utilización

14 Como indica el cuadro 1, las dos variables dependientes presentan mayor desviación típica entre los partidos de Bolivia que entre los de Chile. La distinción entre diputados uninominales y plurinominales del sistema político boliviano es en buena medida responsable de las diferencias constatadas al interior de los partidos. 
de Internet facilita una mayor autonomía del diputado respecto a su partido cuando desarrolla su actividad distrital.

En resumen, todas las variables independientes del modelo, excepto una, contienen capacidad explicativa; además, las relaciones causales se ajustan al sentido esperado. De todo ello se extraen dos conclusiones: ha quedado patente la naturaleza multicausal de los focos de representación; y a pesar del carácter exploratorio del análisis, se ha evidenciado la utilidad del modelo, que combina variables relacionadas con el diputado, con el partido y con el entorno, para comprender el foco de la representación y dónde estas últimas pesan más.

Cuadro 3. Determinantes de focos de representación (análisis de regresión lineal)

\begin{tabular}{lcc}
\hline & $\begin{array}{c}\text { Modelo } 1 \\
\text { El diputado se relaciona } \\
\text { más con el partido }\end{array}$ & $\begin{array}{c}\text { Modelo } 2 \\
\text { Al diputado le influye más el partido } \\
\text { en la toma de decisiones }\end{array}$ \\
\hline Primera legislatura del diputado & 0.089 & $0.209^{*}$ \\
Ideología izquierda-derecha del diputado & $(0.666)$ & $(1.696)$ \\
Importancia del uso de Internet & -0.024 & -0.052 \\
& $(-0.197)$ & $(-0.452)$ \\
Partido en el gobierno & $-0.293^{* *}$ & -0.106 \\
& $(-2.269)$ & $(-0.894)$ \\
Partido con sede en distrito & 0.181 & $0.252^{*}$ \\
Magnitud del distrito & $(1.295)$ & $(1.957)$ \\
Nivel de pobreza en el distrito & 0.242 & $0.249^{*}$ \\
& $(1.618)$ & $(1.804)$ \\
País (Bolivia) & $0.346^{* *}$ & $0.381^{* * *}$ \\
& $(2.622)$ & $(3.129)$ \\
Constante & $-0.484^{*}$ & $-0.510^{* *}$ \\
Número de observaciones & $(-1.899)$ & $(-2.169)$ \\
$R^{2}$ corregida & $0.560^{*}$ & $0.675^{* *}$ \\
\hline
\end{tabular}

Notas: ${ }^{*} p<0.10 ;{ }^{* *} p<0.05 ;{ }^{* \star} p<0.01$. Los valores corresponden a coeficientes beta y los que están entre paréntesis a coeficientes t. Los valores de las dos preguntas de la variable dependiente oscilan en una escala de 1 (sociedad civil) a 10 (partido).

Fuente: Elaboración propia.

\section{Conclusiones}

El objetivo fundamental de esta investigación ha sido aportar evidencia empírica que amplíe el limitado conocimiento sobre la forma de ejercer la representación en América Latina. De las posibles dimensiones del caso, la atención se ha puesto en el foco de la representación, lo que equivale a intentar comprender los intereses que priorizan los parlamentarios en su actividad de representación. 
En particular, se ha analizado el dilema del parlamentario en cuanto a qué intereses debe privilegiar en su labor: los de su partido o los de los ciudadanos de su distrito electoral. Con este fin se ha recurrido a una encuesta entre asesores de diputados de Bolivia y Chile, la cual se concentra en la actividad de representación en los distritos electorales. Hay dos conclusiones principales de este análisis empírico.

En primer lugar, si bien los diputados bolivianos y chilenos prefieren priorizar los intereses ciudadanos del distrito por encima de los del partido, se aprecian diferencias de énfasis significativas entre ellos: los primeros se inclinan más por la representación de los intereses partidistas, en especial los diputados del MAS. Estas diferencias en función del país del diputado reaparecen en la variación al interior de los partidos — más elevada entre los partidos bolivianosDe manera que ciertos factores específicos del contexto de cada país influyen en la preferencia por el foco de la representación.

La segunda conclusión se refiere a las variaciones de las preferencias en los focos de la representación. Dada la diversidad de factores potencialmente explicativos y la escasez de investigación al respecto, se ha realizado un análisis exploratorio que combina factores contextuales, partidistas e individuales. Los resultados muestran la naturaleza multicausal de las preferencias sobre el foco de la representación. La variable país cuenta con mayor poder explicativo, lo que confirma las diferencias señaladas entre los diputados bolivianos y chilenos, así como la incidencia de los factores del contexto nacional. Entre estos, la magnitud de los distritos electorales es muy explicativa y su influencia se ajusta a lo esperado: a mayor magnitud, más focalización en intereses partidistas entre los parlamentarios. Asimismo, se ha comprobado que ciertas características del diputado (carrera política y uso de Internet), junto a rasgos del partido político (presencia en el distrito, ubicación en el gobierno u oposición) y de la circunscripción (nivel de desarrollo) tienen su propio impacto en la priorización de los intereses de representación.

El artículo reivindica el distrito electoral como ámbito de estudio relevante en el análisis empírico de la representación en América Latina que permite, entre otros aspectos, profundizar en la comprensión de los vínculos entre representantes y representados, y problematizar sobre el papel de los partidos políticos en dicha relación. Ahondar en esta línea puede ayudar a comprender los dilemas de dicha actividad en la región y en sus determinantes. 


\section{Referencias}

Adams, J. (2010). Party Competition and Responsible Party Government. Ann Arbor: Michigan University Press.

Alcántara Sáez, M. (2004). ¿Instituciones o máquinas ideológicas? Origen programa y organización de los partidos politicos latinoamericanos. Barcelona: ICPS.

Amaral, S. \& Stokes, S. (2005). La democracia local en Argentina: Clientelismo, capital social e innovación en Argentina. Buenos Aires: Universidad Tres de Febrero.

Andeweg, R. B. \& Thomassen, J. J. A. (2005). Modes of Political Representation: Toward a New Typology. Legislative Studies Quarterly, 30(4), 507-528.

Ansolabehere, S. \& Jones, P. E. (2011). Dyadic Representation. En Schickler, E. \& Lee, F. E. (Eds.). Oxford Handbook of The American Congress (pp. 293-314). Nueva York, Oxford University Press.

Barker, D. \& Carman, C. J. (2010). Yes WE Can or Yes HE Can? Citizen Preferences Regarding Styles of Representation and Presidential Voting Behavior. Presidential Studies Quaterly, 40(3), 431-448.

Barreda, M. \& Ruiz, L. M. (2014). Una Aproximación al Estudio de la Actividad Distrital de los Diputados: los Casos de Chile y Bolivia. Debates, 8(1), 81-103.

Bengtsson, A. \& Wass, H. (2010). Styles of Political Representation: What Do Voters Expect? Journal of Elections, Public Opinion and Parties, 20(1), 55-81.

Brack, N., Costa, O. \& Pequito, C. (2012). Attitudes Towards the Focus and Style of Political Representation among Belgian, French and Portuguese Parliamentarian. Representation, 48(4), 387-402.

Brusco, V., Nazareno, M. \& Stokes, S. (2004). Vote-Buying in Argentina. Latin American Research Review, 39(2), 66-88.

Carman, C. J. (2007). Assessing Preferences for Political Representation in the US. Journal of Elections, Public Opinions and Parties, 17(1), 1-19.

Carroll, R. \& Shugart, M. S. (2007). Neo-Madisonian Theory and Latin American Institutions. En Munck, G. L. (Ed.). Regimes and Democracy in Latin America (pp. 51-101). Oxford: Oxford University Press. 
Contreras, G. (2012). Redes de intermediación política en Chile: Retomando la discusión sobre los brokers políticos en el Chile post-autoritario. Ponencia presentada en el IV Congreso Uruguayo de Ciencia Política, Montevideo, Uruguay.

Converse, P. E. \& Pierce, R. (1979). Representative Roles and Legislative Behavior in France. Legislative Studies Quaterly, 4(4), 525-562.

Corral, M., Sánchez, F. \& Rivas, C. (2016). The Impact of Mixed-member Districts on Legislator's Behavior: The Case of Bolivia. Latin American Politics and Society, 58(1), $29-48$.

Cox, G. (2004). La coordinación estratégica de los sistemas electorales del mundo: hacer que los votos cuenten. Barcelona: Gedisa.

Davidson, R. H., Oleszek, W. J., Lee, F. E. \& Schickler, E. (2014). Congress and Its Members. Washington, DC: Sage/CQ Press.

Deschouwer, K. \& Depauw, S. (2014). Representing the People: A Survey among Members of Statewide and Substate Parliaments. Oxford: Oxford University Press.

Díaz Rioseco, D., Giannini, P., Luna, J. P. \& Núñez, R. (2006). El secreto de mi éxito. Seis caminos para llegar y permanecer en Valparaíso. Revista de Ciencia Politica, 26(1), 69-190.

Do Alto, H. \& Stefanoni, P. (2009). El mas: las ambivalencias de la democracia corporativa. Ponencia en el Coloquio pNud "Democracia interna en la elección de candidatos del Movimiento al Socialismo". La Paz, Bolivia.

Domingo, P. (2006). Bolivia. Fin de un ciclo y nuevas perspectivas politicas (1993-2003). Barcelona: Edicions Bellatera.

Eulau, H., Wahlke, J. C., Buchanan, W. \& Leroy, C. Ferguson, L. C. (1959). The Role of the Representative: Some Empirical Observations on the Theory of Edmund Burke. American Political Science Review, 53(3), 742-756.

Fenno, R. F. (1978). Home Style. House Members in Their Districts. Boston: Little, Brown.

Fiorina, M. P. (1981). Retrospective Voting in American National Elections. New Haven: Yale University Press.

Fiorina, M. P. (1977). Congress: Keystone of the Washington Establishment. New Haven: Yale University Press. 
García Montero, M. \& Marenghi, P. (2008). The Puzzle of Representation: The Behavior of Latin American Legislators and the Interests they Defend. En Alcántara, M. (Ed.). Politicians and Politics in Latin America. Boulder, CO: Lynne Rienner Pub.

Gauja, A. (2013). The Politics of Party Policy: From Members to Legislators. Houndmills: Palgrave Macmillan.

Gibson, R., Margolis, M., Resnick, D. \& Ward, S. J. (2003). Election Campaigning on the WWW in the USA and UK. A Comparative Analysis. Party Politics, 9(1), 47-75.

Luna, J. P. (2014). Segmented Representation. Political Party Strategies in Unequal Democracies. Oxford: Oxford University Press.

Luna, J. P. (2010). Segmented Party-Voter Linkages in Latin America: The Case of the UdI. Journal of Latin American Studies, 42(2), 325-356.

Luna, J. P. \& Mardones, R. (2010). Chile: Are the Parties Over? Journal of Democracy, 21(3), 107-121.

Mainwaring, S. (1999). Party Systems in the Third Wave of Democratization. The Case of Brazil. Stanford: Stanford University Press.

Mainwaring, S., Bejarano, A. M. \& Pizarro, E. (Eds.) (2006). The Crisis of Democratic Representation in the Andes. Standford: Standford University Press.

Mansbridge, J. (2011). Clarifying the Concept of Representation. American Political Science Review, 105(3), 621-630.

Mansbridge, J. (2003). Rethinking Representation. American Political Science Review, 97(4), 515- 528 .

Martínez, A. (2004). La representación política y la calidad de la democracia. Revista Mexicana de Sociología, 66(4), 661-710.

Mayhew, D. (1974). Congress: The Electoral Connection. New Haven: Yale University Press.

Mayorga, R. A. (2008). Populism in Bolivia: Can a Social Movement Govern without a Party? En Woodrow Wilson Center, recuperado el 28 de julio de 2014, de http://www.wilsoncenter .org/sites/default/files/lap_policy_Bolivia1.pdf

Méndez-Lago, M. \& Martínez, A. (2002). Political Representation in Spain: An Empirical Analysis of the Perception of Citizens and MPs. Journal of Legislative Studies, 8(1), 63-90. 
Mitnick, B. M. (2013). Origin of the Theory of Agency: An Account By One of the Theory's Originators. Paper de SSRN, recuperado el 28 de julio de 2014, de http://ssrn.com/abstract=1020378

Offe, C. \& Preuss, U. (1990). Instituciones democráticas y recursos morales. Isegoría, (2), 45-74.

Padró-Solanet, A. \& Cardenal, A. S. (2009). Partidos y política en Internet: Un análisis de los websites de los partidos políticos catalanes. IDP. Revista de Internet, Derecho y Política, (6), 46-61.

Pitkin, H. F. (1967). The Concept of Representation. Los Ángeles, CA: University of California Press.

Rehfeld, A. (2009). Representation Rethought: On Trustees, Delegates, and Gyroscopes in the Study of Political Representation and Democracy. American Political Science Review, 103(2), 214-230.

Ruiz Rodríguez, L. M (2007). Partidos y coherencia. Parlamentarios en América Latina. Madrid: Centro de Estudios Políticos y Constitucionales.

Siavelis, P. \& Morgenstern, S. (Eds.) (2008). Pathways to Power: Political Recruitment and Candidate Selection in Latin America. University Park: Penn State University Press.

Stokes, S. C., Dunning, T., Nazareno, M. \& Brusco, V. (2013). Brokers, Voters and Clientelism. Cambridge: Cambridge University Press.

Szwarcberg, M. (2013). The Microfoundations of Clientelism. Lessons from the Argentina Case. Latin American Research Review, 48(2), 32-54.

Szwarcberg, M. (2012). Uncertainty, Political Clientelism, and Voter Turnout in Latin America: Why Parties Conduct Rallies in Argentina. Comparative Politics, 45(1), 88-106.

Thomassen, J. A. (1994). Empirical Research into Political Representation: Failing Democracy or Failing Models. En Jennings, M. K. \& Mann, T. E. (Eds.). Elections at Home and Abroad: Essays in Honor of Warren Miller (pp. 236-265). Ann Arbor: University of Michigan Press.

Valenzuela, A. (1977). Political Brokers in Chile: Local Government in a Centralized Polity. Durham: Duke University Press.

Weßels, B. (2007). Political Representation and Democracy. En Dalton, R. J. \& Klingemann, H.-D. (Eds.). Oxford Handbook of Political Behavior (pp. 833-849). Oxford: Oxford University Press. 


\section{Anexo}

Distribución de casos en el estudio de los modos de representación en distritos electorales

\begin{tabular}{|c|c|c|c|c|}
\hline Partidos seleccionados & $\begin{array}{l}\text { Diputados en } \\
\text { el Congreso }\end{array}$ & $\begin{array}{l}\text { Diputados } \\
\text { estudiados }\end{array}$ & $\begin{array}{l}\% \text { sobre total } \\
\text { de diputados }\end{array}$ & $\begin{array}{l}\text { Distribución de diputados estudiados } \\
\text { por distritos }\end{array}$ \\
\hline \multicolumn{5}{|l|}{ Bolivia } \\
\hline $\begin{array}{l}\text { Movimiento al Socialismo } \\
\text { (MAS) }\end{array}$ & 88 & 28 & 31.8 & $\begin{array}{l}\text { La Paz (6 diputados), Potosí (7), Cocha- } \\
\text { bamba (5), Pando (1), Beni (2), Chuqui- } \\
\text { saca (2), Tarija (2) y Santa Cruz (3) }\end{array}$ \\
\hline $\begin{array}{l}\text { Plan Progreso para Bolivia- } \\
\text { Convergencia Nacional } \\
\text { (PPB-CN) }\end{array}$ & 37 & 15 & 40.5 & $\begin{array}{l}\text { Chuquisaca (3), Cochabamba (3), La } \\
\text { Paz (1), Santa Cruz (7) y Pando (1) }\end{array}$ \\
\hline Unidad Nacional (UN) & 3 & 2 & 66.7 & La Paz (2) \\
\hline Alianza Social (AS) & 2 & 1 & 50 & Potosí (1) \\
\hline \multicolumn{5}{|l|}{ Chile } \\
\hline $\begin{array}{l}\text { Partido Demócrata Cristia- } \\
\text { no (PDC) }\end{array}$ & 19 & 9 & 47.4 & Distritos $8,10,13,15,16,17,44,49$ y 58 \\
\hline $\begin{array}{l}\text { Partido por la Democracia } \\
\text { (PPD) }\end{array}$ & 18 & 9 & 50 & Distritos $9,11,14,17,19,20,22,43$ y 51 \\
\hline Renovación Nacional (RN) & 17 & 9 & 52.9 & Distritos $7,17,21,29,37,41,42,50$ y 51 \\
\hline $\begin{array}{l}\text { Unión Demócrata Indepen- } \\
\text { diente (UDI) }\end{array}$ & 39 & 2 & 5.1 & Distritos 43 y 49 \\
\hline
\end{tabular}

Fuente: Encuesta de Asesores de Diputados (2013).

Recibido el 7 de septiembre de 2015. Aceptado el 7 de julio de 2016. 